\section{Judgment of angle size: An experimental appraisal}

\author{
IAIN E. MACLEAN and BARRIE G. STACEY \\ University of Strathclyde, Glasgow, C. 1, Scotland
}

Previous work on the estimation of angle size suggested that three factors play a significant part in the forming of an accurate estimation: the size of the angle, its orientation, and the mode in which the response is made. An examination of the judgment by $60 \mathrm{Ss}$ of 12 angles under varied conditions of orientation, response mode, and cue was carried out with the result that the importance of size and orientation was confirmed. Despite an overall significant difference in estimation between response modes, there was no indication of a consistent effect across angles for the different modes. Predictions on the influence of orientation made by Fisher and Beery were tested, and while the former investigator was adequately supported, the latter was held to have reported results on a limited sample of angles which do not generalize to the wider range of angles used in the present study.

Interest in the problem of describing and explaining the characteristic over- and underestimation of the size of angles has been renewed with papers by Beery (1968) and Fisher (1969). Until these studies, the conventional view, stemming from Helmholtz, Titchener, Wundt, Ladd, and Woodworth, that acute angles are overestimated and obtuse angles underestimated was generally accepted. Piaget (1969) found himself able to theorize about the processes underlying this phenomenon, and Campbell (1969), in a discussion of the carpentered-world hypothesis, felt able to cite the 19th century "optical illusion literature" to support his finding on the perceptual distortion of parallelograms, which he described as "normalization" of angles.

Beery (1968) has noted a contradiction between Jastrow (1892) and the early investigators mentioned above, Jastrow alone proposing the view that acute angles are underestimated and obtuse overestimated. The explanation of the under-/overestimation paradox favored by Beery relates the variation in experimental results to the orientation of the stimulus angles. Following an observation by Piaget, Beery generated a hypothetical process determining the judgment of angles which depends upon the relationship of their arms to the conventional Cartesian axes. His objective test of this explanation of angular distortion consisted of the drawing of two "critical" angles by some $20 \mathrm{Ss}$ using matched reproduction. His results give support to his argument about the operation of a form of contour repulsion from Cartesian axes.

Noting the same contradiction as Beery, Fisher (1969) proceeded in three separate experiments to reexamine the question of judgments of acute and obtuse angles. He centered his explanation on the differing results obtained with Ss using either of two chosen response modes: (1) verbal production by drawing after a numerical instruction. In a systematic analysis of the process of reproduction by drawing, as used in Jastrow's study, Fisher argued that a two-stage process takes place. First, the perceiver makes an implicit numerical estimate of angular size then, secondly, constructs an angle to match this. Any reproduced angle will be the resultant of the two judgment biases and will thus yield an ambiguous result which may either maximize or minimize the effect of these biases, depending on whether or not they operate in a common direction. Fisher produced a hybrid curve from his separate studies, employing verbal estimation and angle production which agrees tolerably well with that in Jastrow's paper. An additional finding in Fisher's examination concerned angles located such that they were bisected by the imaginary vertical axis. These are underestimated relative to those bisected by the imaginary horizontal axis. In citing this as a factor justifying the assumption of regular distortions of angular size, he describes it as a systematic tendency, and it appears consistent in the figure which he presents (Fisher, 1969, Fig. 4, p. 60). This demonstration provides a firm generalization for replication, not only within the response mode which Fisher used, namely verbal estimation, but also across alternative response modes.

\section{DESIGN}

The present writers decided to determine the significance of previously noted variables in a single experiment on the judgment of angles in which both their main effects and their interactions could be examined. This was attempted by the use of a large number of Ss in a repeated measures factorial design. The variables studied in the present experiment are most estimation of angle size and (2) angle easily described in point form as follows.

(1) A selection of 12 angles was made, 1 chosen at random from each of the 15-deg bands between 0 and $180 \mathrm{deg}$. There were $8,23,40,47,64,82,99,116,135,148$, 153 , and $171 \mathrm{deg}$.

(2) Each of these angles was presented to the Ss in each of three orientations defined by the position of the bisector (assumed) of the angle. These lay along the Cartesian axes or the four quadrant diagonals at $45 \mathrm{deg}$ to them. A randomizing procedure determined whether the vertex was up/down, right/left, or a combination of these for diagonally presented angles. Fisher (1969) had reported that the direction of the vertex had no effect on judgments obtained in his situation.

(3) In addition, each angle was represented in each of two cue conditions. The multiple-cue condition consisted of the angle lying within a circle with a square containing perpendicular bisectors of each side superimposed outwith the circle (see Fig. 1). The minimal-cue condition consisted of the angle's being presented on a uniform opalescent field.

(4) The Ss were required to judge the stimulus angles using two response modes: first, "as accurate as possible," a numerical estimation of the size, in degrees, of each angle, and secondly, a reproduction of the angle seen, by attaching the second arm of the presented angle, on a response sheet, to one randomly chosen arm drawn at an orientation identical to that on the screen. These tasks were carried out with the stimulus still in view. The two judgments were not obtained successively.

\section{PROCEDURE}

The 72 possible different angle stimuli, 36 (12 angles by 3 orientations) multiple-cue and 36 minimal-cue, were drawn in black on white card and reproduced to give two complete sets on monochromatic projection slides. The sequence of each set of 36 (multiple-cue

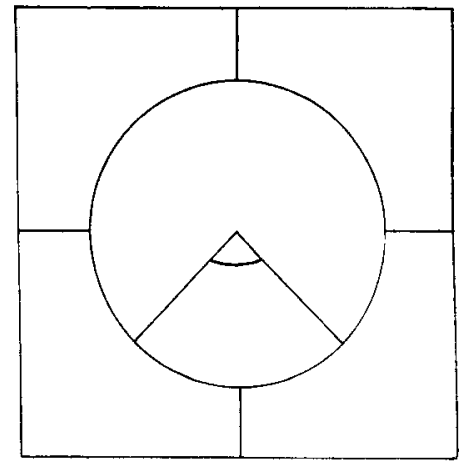

Fig. 1. A vertically oriented angle under multiple-cue conditions. 
Table 1

Analysis of Variance

\begin{tabular}{|c|c|c|c|c|}
\hline Source & ss & df & $\mathrm{ms}$ & $\mathrm{F}$ \\
\hline Subjects (S) & 43159.00 & 59 & 731.51 & - \\
\hline Response Mode (R) & 4138.94 & 1 & 4138.94 & $8.64^{*}$ \\
\hline$R \times S$ & 28259.20 & 59 & 478.97 & - \\
\hline Cue Conditions (C) & 2002.96 & 1 & 2002.96 & $9.64^{*}$ \\
\hline $\mathrm{C} \times \mathrm{S}$ & 12265.06 & 59 & 207.88 & - \\
\hline Orientation (O) & 24069.60 & 2 & 12034.8 & $70.80^{*}$ \\
\hline $\mathrm{O} \times \mathrm{S}$ & 20059.60 & 118 & 169.99 & - \\
\hline Angles (A) & 67919.60 & 11 & 6174.51 & $25.51 *$ \\
\hline$A \times S$ & 157119.40 & 649 & 242.09 & - \\
\hline $\mathrm{R} \times \mathrm{C}$ & 811.56 & 1 & 811.56 & 4.14 \\
\hline $\mathrm{R} \times \mathrm{C} \times \mathrm{S}$ & 11560.26 & 59 & 195.94 & - \\
\hline $\mathrm{R} \times \mathrm{O}$ & 1477.44 & 2 & 738.72 & $5.69 *$ \\
\hline $\mathrm{R} \times \mathrm{O} \times \mathrm{S}$ & 15326.04 & 118 & 129.88 & - \\
\hline $\mathbf{R} \times \mathbf{A}$ & 31603.60 & 11 & 2873.05 & $15.31^{*}$ \\
\hline $\mathbf{R} \times \mathbf{A} \times \mathbf{S}$ & 121804.00 & 649 & 187.68 & - \\
\hline $\mathrm{C} \times \mathrm{O}$ & 669.66 & 2 & 334.83 & 3.10 \\
\hline $\mathrm{C} \times \mathrm{O} \times \mathrm{S}$ & 12758.34 & 118 & 108.12 & - \\
\hline $\mathrm{C} \times \mathrm{A}$ & 5913.10 & 11 & 537.55 & $5.55^{*}$ \\
\hline $\mathrm{C} \times \mathrm{A} \times \mathrm{S}$ & 62912.80 & 649 & 96.94 & - \\
\hline $\mathrm{O} \times \mathrm{A}$ & 31148.40 & 22 & 1415.84 & $15.68 *$ \\
\hline$O \times A \times S$ & 117.209 .20 & 1298 & 90.30 & - \\
\hline $\mathrm{R} \times \mathrm{C} \times \mathrm{O}$ & 738.97 & 2 & 369.48 & 3.54 \\
\hline$R \times C \times O \times S$ & 12331.56 & 118 & 104.50 & - \\
\hline $\mathrm{R} \times \mathrm{C} \times \mathrm{A}$ & 3955.14 & 11 & 359.56 & $3.92^{*}$ \\
\hline$R \times C \times A \times S$ & 59464.80 & 649 & 91.63 & - \\
\hline$R \times O \times A$ & 15869.76 & 22 & 721.35 & $7.58^{*}$ \\
\hline$R \times O \times A \times S$ & 123489.60 & 1298 & 95.14 & - \\
\hline $\mathrm{C} \times \mathrm{O} \times \mathrm{A}$ & 4175.54 & 22 & 189.80 & $2.31^{*}$ \\
\hline $\mathrm{C} \times \mathrm{O} \times \mathrm{A} \times \mathrm{S}$ & 106766.00 & 1298 & 82.25 & - \\
\hline $\mathrm{R} \times \mathrm{C} \times \mathrm{O} \times \mathrm{A}$ & 6060.04 & 22 & 275.46 & $3.13^{*}$ \\
\hline $\mathrm{R} \times \mathrm{C} \times \mathrm{O} \times \mathrm{A} \times \mathrm{S}$ & 114300.00 & 1298 & 88.06 & - \\
\hline Total & 1219339.18 & & & \\
\hline
\end{tabular}

*Significant at or beyond the $1 \%$ level.

matched reproduction, $\mathrm{MuC} / \mathrm{MR}$; multiple-cue verbal estimation, $\mathrm{MuC} / \mathrm{VE}$; minimal-cue matched reproduction, MiC/MR; minimal-cue verbal estimation, $\mathrm{MiC} / \mathrm{VE}$ ) was internally randomized and two orders of presentation of the complete sets decided. These were $\mathrm{MuC} / \mathrm{VE}$, $\mathrm{MuC} / \mathrm{MR}, \mathrm{MiC} / \mathrm{VE}, \mathrm{MiC} / \mathrm{MR}$, and $\mathrm{MiC} / \mathrm{VE}$, $\mathrm{MiC} / \mathrm{MR}, \mathrm{MuC} / \mathrm{VE}, \mathrm{MuC} / \mathrm{MR}$.

A total of $60 \mathrm{Ss}$ was used, a heterogeneous sample of male and female, working- and middle-class adults with no previous knowledge of perceptual research Each $S$ was paid a fee for cooperating in the study. Testing was done in five group sessions. First, the word "angle" was defined and a number of illustrations were provided, covering the range from 0 to $180 \mathrm{deg}$. The Ss were then presented with each set of 36 slides at the rate of one $/ 15 \mathrm{sec}$ for verbal estimation and one $/ 25 \mathrm{sec}$ for matched reproduction. Interset breaks of $5 \mathrm{~min}, 15 \mathrm{~min}$ (for coffee), and $5 \mathrm{~min}$ were given to avoid boredom. Illumination within the test room was just adequate for writing but was reduced around the $17 \times 22$ in. side-projection screen on which the stimuli were presented. The screen, which was rectangular with rounded corners, was vertical to the line of vision and presented an angle with arms 6 in. in length. No $S$ was less than $6 \mathrm{ft}$ from the screen and none more than $20 \mathrm{ft}$. Responses were recorded on prepared sheets which had the number of appearance of each slide alongside the space for response. As each slide was projected, the $E$ called out the number of the slide.

Matched reproduction drawings were scored by means of a protractor; these scores plus the numerical estimates were converted to exact error scores by subtraction of the actual size of the stimulus angle.

\section{RESULTS}

The error scores were subjected to an analysis of variance using a four-way repeated measures design. Examination of the analysis of variance results in Table 1 reveals the orientation effect as significant beyond the $1 \%$ level. The value of the $F$ ratio is 70.80 with $\mathrm{df}=2 / 118$. For each orientation the means obtained were: (1) vertical, -3.79 , (2) diagonal, -1.13 , and (3) horizontal, 0.23 .

Figure 2 represents the overall error scores for each of the 12 stimulus angles, for which the Fratio of 25.51 , with $\mathrm{df}=11 / 649$, proved significant beyond the $1 \%$ level.

An F ratio of 15.68 , with $d f=22 / 1298$, was obtained for the Orientation by Angles interaction $(p<.01)$. This interaction is represented graphically in Fig. 3.

For the Response Mode by Orientation interaction, an $F$ ratio of 5.69 , with $\mathrm{df}=2 / 118$, was found $(\mathrm{p}<.01)$. The

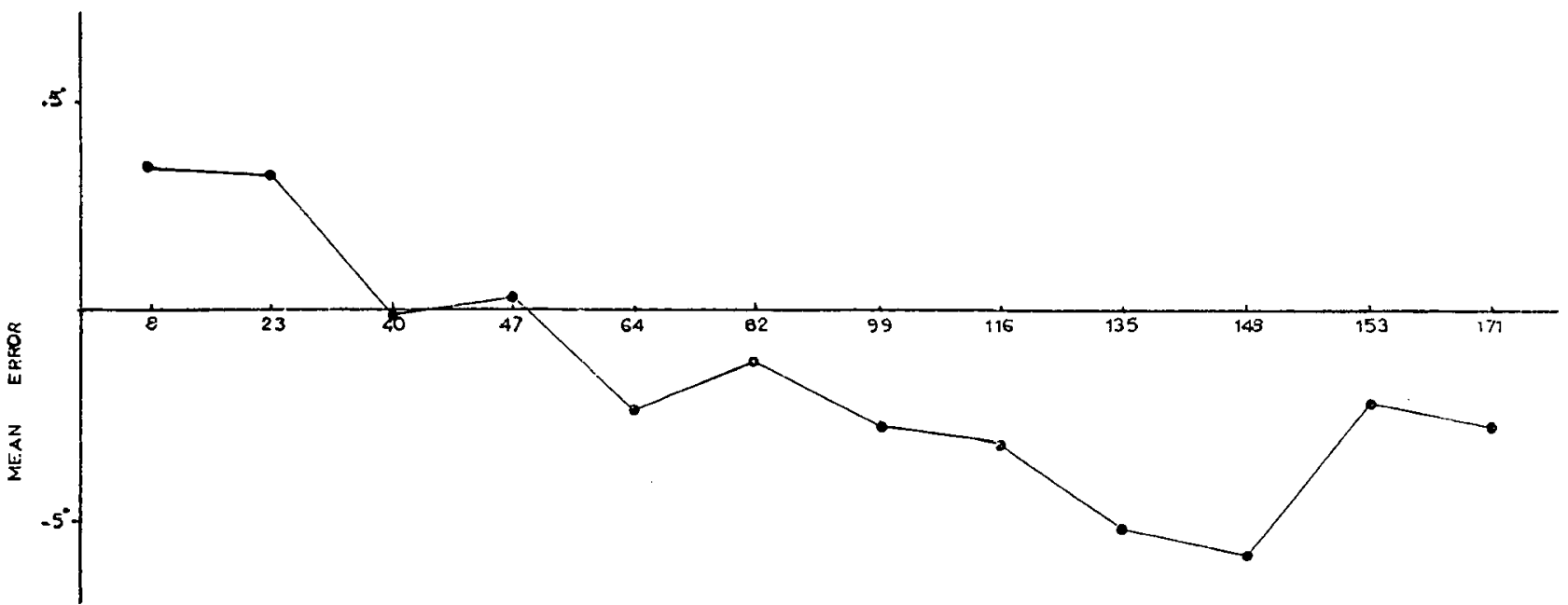

Fig. 2. Overall mean error score for each angle presented. 


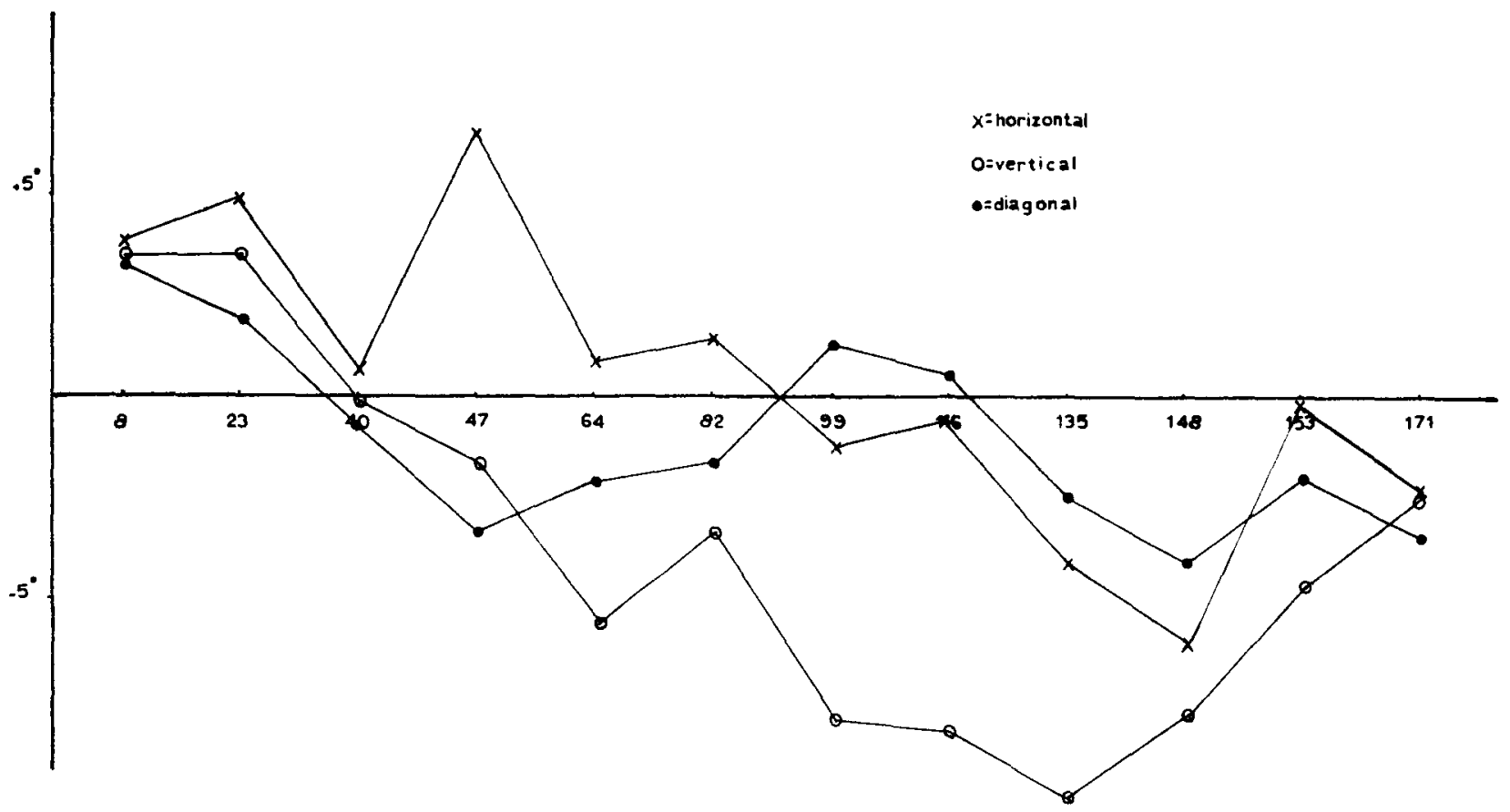

Fig. 3. Mean error scores in the Orientation by Angles interaction.

alues comprising this interaction are:

$\begin{array}{cc}\text { Matched } & \text { Verbal } \\ \text { Reproduction } & \text { Estimation }\end{array}$

$\begin{array}{llr}\text { Jertical } & -3.99 & -3.50 \\ \text { Jiagonal } & -1.82 & -0.47 \\ \text { Jorizontal } & -0.98 & 1.45\end{array}$

With $\mathrm{df}=1 / 59$, the $\mathrm{F}$ ratio of 8.64 sbtained for the difference between the nain effects of verbal estimation and matched reproduction (error scores of -0.87 and -2.25 , respectively) is significant at the $1 \%$ level.

The Response Mode by Angles interaction yields an $F$ ratio of 15.31 , with $\mathrm{df}=11 / 649$, which is significant beyond the $1 \%$ level. Table 2 sets out the mean error scores relevant to this interaction.

An $F$ ratio of 9.64 , with $\mathrm{df}=1 / 59$, was obtained for the main effect of cue conditions $(p<.01)$. Multiple-cue conditions gave a mean error of -2.04 and minimal-cue, one of -1.08 .

Further interactions which were significant at or beyond the $1 \%$ level are: Cue Conditions by Angles, Response Mode by Orientation by Angles, Response Mode by Cue Conditions by Angles, Cue Conditions by Orientations by Angles, and
Response Mode by Cue Conditions by Orientations by Angles.

\section{DISCUSSION}

The most marked effect found in this study concerns the prediction made from Fisher's work (1969), namely that angles presented in a vertical orientation would be underestimated compared with those presented horizontally. This prediction is clearly upheld and the intermediate orientation, diagonal, yields a mean error score between the other two conditions. Looking at the interaction between orientation and angles in Fig. 3, we find nothing to contradict Fisher's (1968) assertion that "all sideways-pointing angles are judged to be greater in size than similar angles which point upwards and downwards." Indeed, the fact that a significant interaction occurs reflects the unstable effect of diagonal presentation with different angles in our range and also the range of variation between "vertical" and "horizontal" judgments of the same angles. This latter overall relationship remains constant, but the absolute differences are reduced at either end of the range, in comparison with the band of 47 to $135 \mathrm{deg}$. This corresponds to the position in Fisher's (1969) Fig. 4, with the differences in his case narrowed around $90 \mathrm{deg}$ by the nonperceptual consequences of using 5-deg intervals and a free-estimation response mode (see also Chapanis, 1951). Turning to the Orientation by Response Mode interaction (see results section), the same consistent overestimation of horizontal angles relative to vertical ones may be seen, and there is yet a further indication in that the difference between the two response mode means are least for vertically presented angles, this suggesting that the finding about underestimation of the vertical is a robust one. More generally, the fact that our data on orientation, derived from a group testing situation, are in accord with Fisher's, derived from a more closely controlled individual testing situation, lends further weight to both sets of data.

A specific test of Fisher's assertion, on a basis other than analysis of variance, is contained in Fig. 4 for a situation very similar to the one he used. Diagram A represents the results of the section of our study which differs from Fisher's procedure mainly in respect to the stimulus angles chosen; Fisher's angles were at each 5-deg point from 0 to $180 \mathrm{deg}$, whereas a selection of 12 angles, 1 randomly chosen from each of the $15-\mathrm{deg}$ bands between 0

Table 2

Mean Error Scores for Response Mode by Angles Interaction

\begin{tabular}{|c|c|c|c|c|c|c|c|c|c|c|c|c|}
\hline & $8 \mathrm{Deg}$ & 23 Deg & $40 \mathrm{Deg}$ & 47 Deg & $64 \mathrm{Deg}$ & $82 \mathrm{Deg}$ & $99 \mathrm{Deg}$ & $116 \mathrm{Deg}$ & 135 Deg & $148 \mathrm{Deg}$ & 153 Deg & $171 \mathrm{Deg}$ \\
\hline Matched Reproduction & 4.61 & 3.30 & 0.34 & 0.04 & -2.48 & -2.04 & -0.91 & -1.65 & -6.06 & -9.58 & -6.09 & -6.52 \\
\hline Verbal Estimation & 2.44 & 3.39 & -0.61 & 0.57 & -2.25 & -0.57 & -4.56 & -4.65 & -4.21 & -2.36 & 1.40 & 0.99 \\
\hline
\end{tabular}



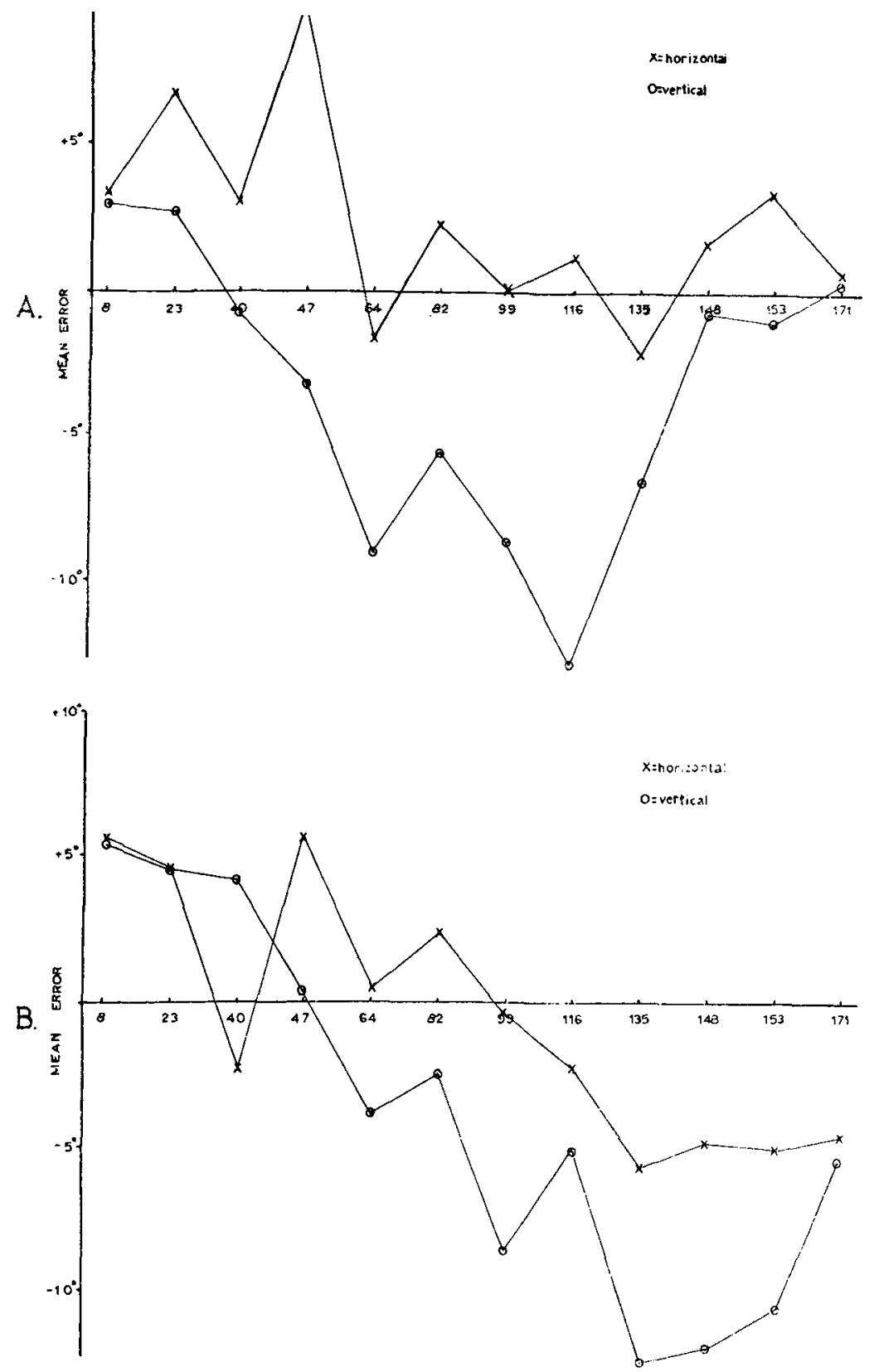

Fig. 4. Mean error scores with each angle presented in minimal-cue conditions and with: (A) verbal estimation response mode; (B) matched reproduction response mode.

and $180 \mathrm{deg}$, constituted the stimuli in the present study. It can be seen that the error scores obtained fully support "vertical" underestimation, compared with "horizontal." Diagram B, which represents the situation altered to the extent of substituting matched reproduction as the response mode in place of verbal estimation, also confirms the previous finding in all angles studied, save one. That this is so, despite a significant change in response mode, attests to the stability of the relationship.

The relevant second order interactions yield little further information on the remains below both the other curves (i.e., diagonally presented angles are underestimated relative to the other orientations), and from 99 to $153 \mathrm{deg}$, it remains above the other two orientations. On this point we merely note that $82 \mathrm{deg}$ is the largest angle used which, with diagonal presentation, is contained wholly within one quadrant, while $99 \mathrm{deg}$ is the least angle to span three quadrants.

With so many interacting variables, a significant $F$ ratio for the overall differences among angles may not, in fact, reflect a firm trend over the 0 - to 180-deg range when smaller combinations of variables are operating. Perhaps the most acceptable conclusion from the graphical representation in Fig. 2 is that there exists a gradual decline in estimated size from 8 to $148 \mathrm{deg}$, with a reversal over the final two angles, 153 and $171 \mathrm{deg}$, presented. A more wide-ranging conclusion would be subject to counterexamples within subsets of data. Of more significance is the bearing of our data on the hypothesized overestimation of acute and underestimation of obtuse angles so prominent in the literature. For angles presented in the horizontal (but not diagonal or vertical) orientation, this relationship holds, as is clear from examination of the Orientation by Angles interaction presented in Fig. 3. This relationship for the horizontal orientation is upheld on examination of the second-order interactions, where the only sizable discrepancy occurs for the two angles, 153 and $171 \mathrm{deg}$, with verbal estimation. That this over-/underestimation trend holds with matched reproduction (Jastrow's response mode) in the Response by Orientation by Angles interaction for horizontally, but not diagonally or vertically, presented angles suggests that fortuitous choice of angle sizes and experimental conditions were, as much as anything, responsible for the contradiction between Jastrow and other early investigators. Another coincidental similarity of results is illustrated by Fig. 5 , where the curve for the diagonal orientation with matched reproduction in the present study can be seen, along with the differently derived curve published by Jastrow and Fisher's hybrid curve predicting Jastrow's results. While Fisher has performed a service by drawing attention to the question of response mode, the logical steps he took to obtain his hybrid curve are less parsimonious than making the "specific experimental conditions" assumption suggested above.

If we consider next the effect of response mode on judgments obtained, we first note that the overall effect is for matched reproduction to exhibit greater 


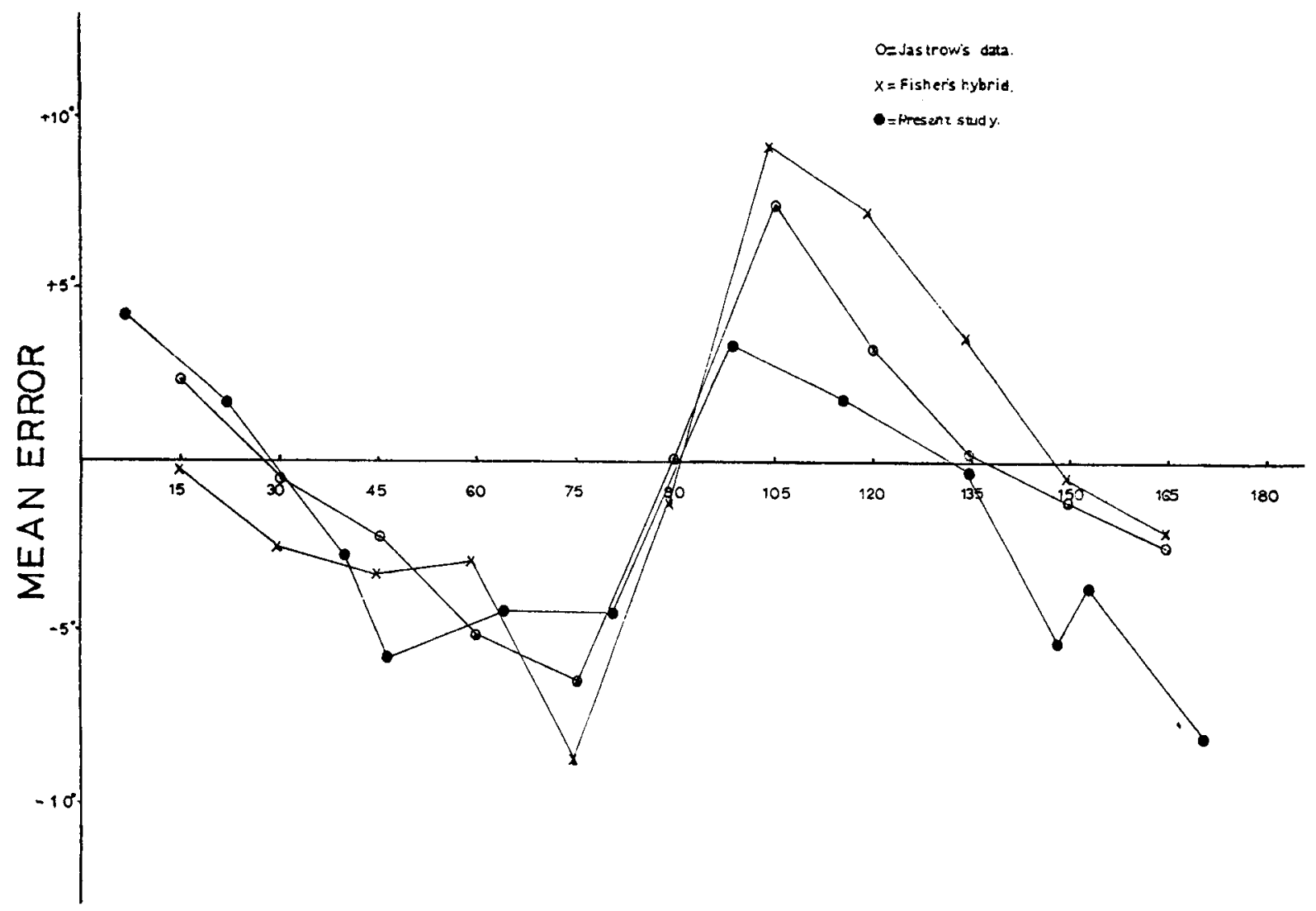

Fig. 5. Curves derived from the data of the present study and the previously published studies of Jastrow and Fisher.

underestimation. Secondly, Table 2, showing the significant interaction between response mode and angles, yields the conclusion that this overall difference is due to the final three angles, 148,153 , and $171 \mathrm{deg}$. These two findings are present to the same degree in both the significant second-order interactions, including response mode, and indeed, as noted in a preceding section, it is the overestimation of the final two angles with the verbal response mode which disturbed an otherwise consistent pattern in the acute/obtuse misestimation example. To account for the differences within the Response Mode by Angles interaction, it could be postulated that the Ss, while approaching the limits of the range given when verbal estimates were called for (perhaps displaying some subjective expected probability of occurrence of elements throughout the range), did not perform similarly using matched reproduction. It may be that a perceptuomotor process causes any angle perceived to be minimally different from a straight line to be reproduced markedly different from a straight line.

Perhaps the least explicable of all the results in our analysis of variance table is that relating to cue conditions. This variable had been included partially because of the possible relevance of findings on angular judgment in an applied situation and partially to allow for an examination (to appear in a subsequent paper) of Gogel's (1968) work on calibration equations. From the significant interactions in which cue conditions are present, the conclusion must be that there is a small, but significant, difference in estimation through the range that disturbs the trend of interacting variables only with the 148-deg angle, which seems substantially underestimated whenever it is presented in multiple-cue conditions. Graphical examination of the significant third-order interaction (Angles by Orientation by Position by Cue Conditions) suggests that the underestimation of $148 \mathrm{deg}$ under multiple-cue conditions contributed substantially to the previously noted dependence of underestimation with matched reproduction on the final three angles. Indeed, in no case where a multiple-/minimal-cue comparison can be made within the third-order interaction is there a reversal of the finding that $148 \mathrm{deg}$ is underestimated to a greater extent under multiple- rather than minimal-cue conditions.

Beery's (1968) assumption that the spatial orientation of an angle stimulus influences judgment of angle size seems, given the evidence of Fisher and the present writers, wholly reasonable. But when one scrutinizes his development of this premise, then a further less favorable conclusion emerges. Using Beery's contour repulsion/attraction model, we have been able to generate predictions applicable in the various subsections of our study where matched reproduction was utilized, as in Beery's study. The six combinations of matched reproduction, orientation, and cue condition produce 72 angle stimuli on which to test Beery's predictions. Each angle is the mean of the judgments of 60 Ss. Table $3 \mathrm{~A}$ indicates the predictions made in general terms, and Table $3 \mathrm{~B}$ shows that when these were tested, it was found that in only 24 out of 72 cases were Beery's predictions upheld. Under only one condition out of six did his predictions yield more positive than negative results, and that by the slim ratio of $7: 5$. This condition was matched reproduction of horizontally oriented angles presented on a minimal-cue field. Beery's field, however, was anything but minimal-cue, with clearly bounded paper and sufficiently small distances that parallel-matching (Helmholtz, 1910, p. 173) might even have been an operating factor. In the conditions nearest to Beery's in our study (i.e., 
Table 3

Error in the Present Study Using Matched Reproduction, as Predicted from Beery's Analysís of Angular Estimation

\section{A. Predictions \\ For horizontal or vertical orientation:}

0 Deg - 40 Deg Underestimate

41 Deg - 90 Deg Overestimate

$91 \mathrm{Deg}$ - $140 \mathrm{Deg}$ Underestimate

$141 \mathrm{Deg}-180 \mathrm{Deg}$ Overestimate

B.

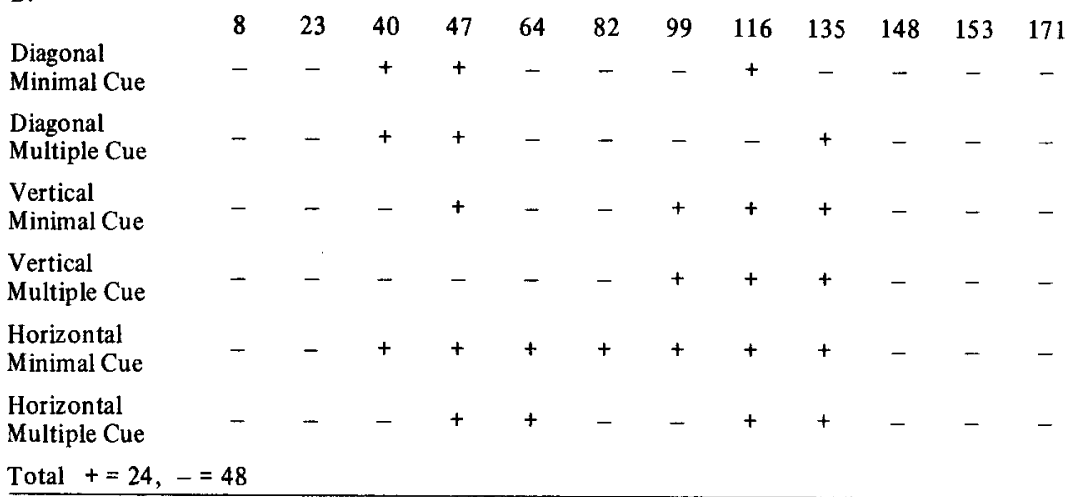

Note-Test of predictions in A for mean error scores of conditions utilizing matched reproduction. Each test based on 60 judgments.

matched reproduction of $64 \mathrm{deg}$ in horizontal orientation and $116 \mathrm{deg}$ in vertical orientation, both with multiple cues), we do, in fact, find that the mean errors are close to those he reported: in the present study +1.52 and $-4.72 \mathrm{deg}$, respectively, corresponding to Beery's results of $+1.1 \mathrm{deg}$ and $-4.0 \mathrm{deg}$. I'he earlier comment on results as fortuitous coincidence of experimental and stimulus conditions applies here more clearly, for it is only within the range around the two angles chosen by Beery that any approximation to a greater-than-chance number of confirmed predictions takes place. That the regions of $0-40 \mathrm{deg}$ and 140-180 deg have far below chance correct

180-deg range.

The findings of this and earlier studies cannot be generalized to more active situations, where an $\mathbf{O}$ can place an angle stimulus in his most favored plane or move it through several positions within a plane A less constricting situation may well produce more accurate estimation, though it would then be very difficult to define orientation in any conventional sense. The passive situation is, of course, the interesting one for practitioners of ergonomics. The next problem to be tackled is the identification of the determinants of the angular distortions reported by Fisher and the present writers, whether or not this is in a situation of theoretical or applied significance (Maclean, 1969; Pollack \& Chapanis, 1952).
0 Deg - 50 Deg Underestimate 51 Deg - 90 Deg Overestimate $91 \mathrm{Deg}$ - 130 Deg Underestimate $131 \mathrm{Deg}$ - 180 Deg Overestimate

\section{REFERENCES}

BEERY, K. E. Estimation of angles. Perceptual \& Motor Skills, 1968, 26, 11-14.

CAMPBELL, D. T. Parallelogram reproduction and the "carpentered world" hypothesis: Suggestions for cross-cultural research. Northwestern University, 1969.

CHAPANIS, A. Studies of manual rotary positioning movements. Journal of Psychology, 1951, 31, 51-71.

FISHER, G. H. The frameworks for perceptual localization. Department of Psychology University of Newcastle upon Tyne, 1968.

FISHER, G. H. An experimental study of angular subtension. Quarterly Journal of Experimental Psychology, 1969, 21, 356-366.

GOGEL, W. C. The measurement of perceived size and distance. In W. D. Neff (Ed.) Contributions to sensory physiology. Vol. 3. New York: Academic Press, 1968.

HELMHOLTZ, H. von Treatise on physiological optics. Vol. III. (1910) Translated by J. P. C. Southall. Optical Society of America, 1925.

JASTROW, J. On the judgment of angles and position of lines. American Journal of Psychology, 1892, 5, 214-248.
MACLLEAN, I. E. Koad works marking lamps. Roads \& Road Construction, 1969, 47, 41-42.

PIAGET, J. The mechanisms of perception. London: Routledge, 1969.

POLLACK, W. T., \& CHAPANIS, A. The apparent length of a line as a function of its inclination. Quarterly Journal of Experimental Psychology, 1952, 4, 170-178.

\section{NOTE}

1. The authors acknowledge the assistance given by Mrs. A. Greenwood and Dr. W. Cheyne in the facilitating of computer analysis of the data and Mr. R, B. Bett in commenting upon an eatly draft of the paper.

(Accepted for publication November 27, 1970.) 